\section{(2)} OPEN ACCESS

\title{
Global incidence and mortality of neonatal sepsis: a systematic review and meta-analysis
}

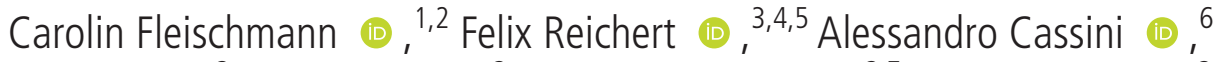 \\ Rosa Horner, ${ }^{3}$ Thomas Harder, $^{3}$ Robby Markwart 다, 3,7 Marc Tröndle (1) , \\ Yoanna Savova, ${ }^{3}$ Niranjan Kissoon (1) , ${ }^{8}$ Peter Schlattmann (1) , ${ }^{9}$ \\ Konrad Reinhart @ $1{ }^{10}$ Benedetta Allegranzi, ${ }^{6}$ Tim Eckmanns ${ }^{3}$
}

- Additional material is published online only. To view, please visit the journal online (http://dx.doi.org/10.1136/ archdischild-2020-320217).

For numbered affiliations see end of article.

\section{Correspondence to} Dr Carolin Fleischmann, Center for Sepsis Control and Care, Jena University Hospital, Jena 07743, Germany;

carolin.fleischmann@med.unijena.de

CF and FR contributed equally.

Received 13 July 2020

Revised 14 December 2020

Accepted 15 December 2020

Published Online First

22 January 2021
Check for updates

(c) Author(s) (or their employer(s)) 2021. Re-use permitted under CC BY. Published by BMJ.

To cite: Fleischmann $C$, Reichert F, Cassini A, et al. Arch Dis Child

2021;106:745-752.

\begin{abstract}
Background Neonates are at major risk of sepsis, but data on neonatal sepsis incidence are scarce. We aimed to assess the incidence and mortality of neonatal sepsis worldwide.
\end{abstract}

Methods We performed a systematic review and meta-analysis. 13 databases were searched for the period January 1979-May 2019, updating the search of a previous systematic review and extending it in order to increase data inputs from low-income and middleincome countries (LMICs). We included studies on the population-level neonatal sepsis incidence that used a clinical sepsis definition, such as the 2005 consensus definition, or relevant ICD codes. We performed a random-effects meta-analysis on neonatal sepsis incidence and mortality, stratified according to sepsis onset, birth weight, prematurity, study setting, WHO region and World Bank income level.

Results The search yielded 4737 publications, of which 26 were included. They accounted for 2797879 live births and 29608 sepsis cases in 14 countries, most of which were middle-income countries. Random-effects estimator for neonatal sepsis incidence in the overall time frame was 2824 (95\% Cl 1892 to 4194) cases per 100000 live births, of which an estimated $17.6 \%$ $9(95 \% \mathrm{Cl} 10.3 \%$ to $28.6 \%)$ died. In the last decade (2009-2018), the incidence was 3930 (95\% Cl 1937 to 7812) per 100000 live births based on four studies from LMICs. In the overall time frame, estimated incidence and mortality was higher in early-onset than late-onset neonatal sepsis cases. There was substantial betweenstudy heterogeneity in all analyses. Studies were at moderate to high risk of bias.

Conclusion Neonatal sepsis is common and often fatal. Its incidence remains unknown in most countries and existing studies show marked heterogeneity, indicating the need to increase the number of epidemiological studies, harmonise neonatal sepsis definitions and improve the quality of research in this field. This can help to design and implement targeted interventions, which are urgently needed to reduce the high incidence of neonatal sepsis worldwide.

\section{INTRODUCTION}

Sepsis is a dysregulated host response to infection leading to life-threatening organ dysfunction. ${ }^{1}$ Although neonates are highly vulnerable to sepsis, incidence estimates for this age group are lacking from many countries. ${ }^{2}$ Recently, the Global Burden

\section{What is already known on this topic?}

- The Global Burden of Disease (GBD) estimated 1.3 million annual incident cases of neonatal sepsis and other infections (approximately 937 cases per 100000 live births) and 203000 sepsis-attributable neonatal deaths. However, important contributors to the burden of neonatal infection and sepsis, such as pneumonia, are not captured by this estimate based on the GBD definition.

- A previous systematic review identified only eight studies on neonatal sepsis incidence from five countries that used a clinical sepsis definition.

\section{What this study adds?}

- We found in 26 studies a pooled neonatal sepsis incidence of 2824 sepsis cases per 100 000 live births (95\% Cl 1892 to 4194) and a mortality of $17.6 \%$ (95\% Cl $10.3 \%$ to $28.6 \%$ ). Preterm and very low birthweight neonates were particularly affected, and there were considerable regional differences in incidence.

- Data are lacking from many countries, underlining the need for further epidemiological research to guide interventions that reduce neonatal incidence and mortality.

of Disease (GBD) Study 2016/2017 estimated 1.3 (95\% CI 0.8 to 2.3 ) million annual incident cases of neonatal sepsis worldwide, ${ }^{3}$ resulting in 203000 (95\% CI 178700 to 267 100) sepsis-attributable deaths. ${ }^{4}$ Neonates are disproportionately affected in low-income and middle-income countries (LMICs) with a high prevalence of infectious diseases ${ }^{5}$ and poor access to adequately equipped and staffed healthcare facilities. ${ }^{6}$ In sub-Saharan Africa alone, an estimated 5.3-8.7 million disability-adjusted lifeyears have been lost in 2014 due to neonatal sepsis and consecutive long-term morbidity. ${ }^{7}$ Neonatal sepsis has resulted in an estimated economic burden of up to US $\$ 469$ billion in this region (2014 data). ${ }^{7}$

In a previous systematic review and meta-analysis, we compiled evidence on the burden of paediatric sepsis including neonatal sepsis. ${ }^{2}$ Only eight studies that used a clinical definition of neonatal 
sepsis could be identified, of which five studies originated from LMICs. We aimed to assess the global incidence and mortality of neonatal sepsis with a particular focus on LMICs by updating and extending this previous systematic review. Secondary objectives were to compile data on underlying organisms and antimicrobial resistance, hospital length of stay and sepsis-attributable mortality.

\section{METHODS}

The protocol of this systematic review was registered in PROSPERO (CRD42020149085).

\section{Search strategy and selection criteria}

As starting point, we used the previously published systematic review on neonatal sepsis incidence. ${ }^{2}$ All studies included in this review were added to our search. We then searched 13 electronic databases: MEDLINE, Embase, LILACS, African Journals Online, OpenGREY, MedCarib, Index Medicus for the WHO Eastern Mediterranean, African, South East Asia and Western Pacific Regions, IndMed, Web of Science and WHOLIS. No language or publication restrictions were applied. We updated the search strategy from the previous systematic review. ${ }^{2}$ Furthermore, we used a comprehensive search strategy that combined a list of sepsis terms with the individual names of LMICs suggested by the Cochrane Effective Practice and Organisation of Care $(\mathrm{EPOC})^{8}$ group to search for studies published between January 1979 and May 2019 (see online supplmental appendix 1). Moreover, we hand-searched reference lists of relevant publications. Studies were eligible for inclusion if they reported population-level incidence or prevalence of neonatal sepsis (neonatal sepsis cases per live births of the hospital or region in a defined time frame). Neonatal sepsis had to be defined according to the International Consensus Conference on Paediatric Sepsis Definitions, ${ }^{9}$ sepsis $1{ }^{10}$ sepsis $2^{11}$ or sepsis $3^{1}$ or modified clinical criteria, such as clinical signs of neonatal sepsis (hypothermia, bradycardia and apnoea) in the presence of infection. Studies based on sepsis-relevant ICD-9/ICD-10 codes were also included. Since definitions varied widely, we accepted definitions of early-onset sepsis (EOS) as sepsis within the first 2-7 days of life. Depending on the EOS definition in each study, late-onset sepsis (LOS) was defined as sepsis diagnosed from 3 to 8 days until 28 days after birth, resulting in individual studies with no double counting, but in overlap between the definitions of EOS and LOS. We excluded studies that solely reported culture-proven sepsis cases, incidence per hospital admissions, or used the definition of possible severe bacterial infections (PSBIs) instead of neonatal sepsis, since neonatal sepsis accounts for only one out of four cases of PSBI. ${ }^{12}$ We excluded studies limited to other subgroups of sepsis, pathogens or specific patient groups, and studies with a missing study methodology description.

\section{Data compilation and risk of bias assessment}

Abstracts and full texts were reviewed by two independent investigators (CF, FR, MT, RH and YS). Discrepancies were resolved by discussion. Non-English articles were assessed by native speakers with medical backgrounds. Data extraction items and strategies can be found in the online supplemental appendix. Pathogen and antibiotic resistance data were only considered from the year 2000 onwards. Authors were contacted to specify methods and to provide additional data. Studies that met the inclusion criteria were assessed for risk of bias by the Hoy tool. ${ }^{13}$

\section{Statistical analyses}

We categorised the studies by sepsis type (EOS, LOS and EOS/ LOS combined). For subgroup analyses, participants were stratified by birth weight into normal birth weight $(\geq 2500 \mathrm{~g})$, low birth weight $(1500-2499 \mathrm{~g})$ and very low birth weight $(<1500 \mathrm{~g})$ neonates, and by gestational age into preterm $(<37$ weeks) and term neonates. Countries were categorised by WHO region and World Bank income level, and studies by communitybased or hospital-based design and decade in which the study was conducted according to the start of their observation period (before 1989, 1989-1998, 1999-2008 and 2009-2018). We conducted meta-analyses of sepsis incidence per 100000 live births, mortality per 100 sepsis cases and the proportion of culture-proven sepsis with $95 \% \mathrm{CI}$ using the package 'meta' V.4.9.5 in R V.3.6.1 (online supplemental appendix 2). We calculated pooled estimates using a random-effects model with variance stabilising logit transformed proportions and estimated between-study variance $\tau^{2}$ using the Sidik-Jonkman estimator. We used $I^{2}$ statistics to quantify statistical heterogeneity.

\section{RESULTS}

We identified 4737 records (figure 1); 250 studies underwent full-text screening; and 26 fulfilled the inclusion criteria. ${ }^{14-39}$

The entire study population comprised 2797879 live births in 14 countries and five WHO regions (online supplemental appendix eTable 1 and figure 2). Two studies originated from low-income countries, 20 from middle-income and four from high-income countries (HICs). Studies were mostly prospective $(\mathrm{n}=18 / 26)$. We included 21 cohort studies, 4 trials and 1 casecontrol study. The majority were single-centre studies. Seven studies used a community-based design; 19 were hospital-based, including studies limited to neonatal intensive care units (NICUs) or studies with entire hospitals under observation. Studies were at moderate to high risk of bias (online supplemental appendix eTable 2).

Overall, 29608 sepsis cases were identified. Sepsis definitions varied by diagnostic criteria and time intervals (online supplemental appendix Table e1).

Incidence of EOS/LOS combined was reported by 21 studies, of which 5 provided separate results for EOS and LOS. EOS and LOS were distinct subgroups in these studies with no overlap. The remaining five studies reported exclusively on EOS. If not stated otherwise, our results refer to studies that report on EOS/ LOS combined. Separate analyses for EOS or LOS are provided if the number of studies was sufficient.

We found a random-effects estimate of 2824 neonatal sepsis cases per 100000 live births (95\% CI 1892 to 4194) in the overall time frame (before 1989-2018), with a 2.6 times higher incidence of EOS (2469/100 000, 95\% CI 1424 to 4250$)$ than LOS (946/100 000, 95\% CI 544 to 1642) (figure 3). For the past decade (2009-2018), the random-effects estimate for EOS/LOS incidence was 3930/100 000 (95\% CI 1937 to 7812) based on four studies from LMICs. Compared with estimates from past decades, we found no clear temporal trends but wide CI for each epoch (1999-2008: 2706, 95\% CI 1451 to 4993; 1989-1998: 4012, 95\% CI: 1808 to 8665 ; before 1989: 1250, 95\% CI 534 to 2896). Of note, studies included in each time stratum differed in terms of study design and country of origin. The estimated incidence of EOS for the decade until 2018 was 3112/100 000 (95\% CI 898 to 10 222) based on four studies (table 1). Only one study reported LOS incidence estimates from the past decade (investigators of Delhi Neonatal Infection Study (DeNIS) collaboration: $658,95 \%$ CI 607 to 713 , per 100000 ).

Birth weight and gestational age were inversely related to EOS incidence, resulting in the highest incidence in VLBW (17 129/100 000, 95\% CI 9192 to 29 679) and preterm neonates 


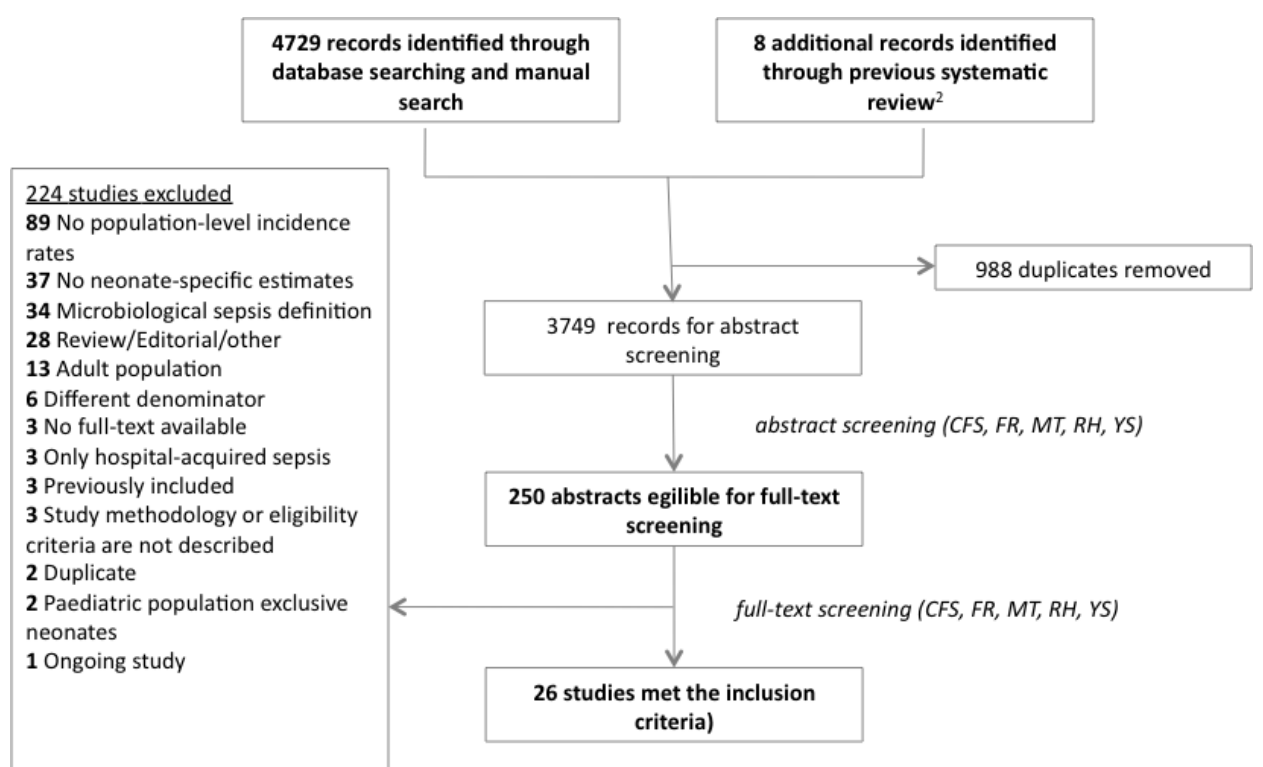

Figure 1 Flow of study inclusion.

(10 252/100 000, 95\% CI 7891 to 13218 ) (online supplemental appendix eFigure 1).

Incidence estimates were higher in community-based studies $(8549 / 100000,95 \%$ CI 5520 to 13011$)$ than in hospitalbased studies (1986/100 000, 95\% CI 1350 to 2914) (online supplemental appendix eFigure 2). Although the 95\% CIs for each income stratum were overlapping, compared with HICs, neonatal sepsis incidence was 1.8 -fold higher in middle-income countries and 3.5-fold higher in low-income countries (online supplemental appendix figure 3). Incidence was highest in studies from Africa (5244/100 000, 95\% CI 2505 to 10651 ; table 1).

We observed substantial heterogeneity between studies, even within subgroup analyses.
Overall, the random-effects estimate for neonatal sepsis case mortality was $17.6 \%$ (95\% CI $10.3 \%$ to $28.6 \%$ ), and $16.4 \%$ (95\% CI 9.8 to 26.1 ) and $9.1 \%$ (95\% CI 2.1 to 32.5$)$ for EOS and LOS, respectively (online supplemental appendix figure 4). Again we observed substantial heterogeneity $\left(\mathrm{I}^{2} \geq 90 \%\right)$. The investigators of DeNIS collaboration attributed $24 \%$ of neonatal deaths to sepsis. ${ }^{18}$

In total, 16 studies reported a proportion of culture-proven sepsis, resulting in a random-effects estimate of $31.8 \%(95 \% \mathrm{CI}$ $23.5 \%$ to $41.4 \%$ ) for EOS/LOS combined (online supplemental appendix figure 5). For EOS, lower proportions were reported $(11.4 \%, 95 \%$ CI 2.7 to $37.1 \%)$ based on six studies than for LOS (27.5\%, 95\% CI 8.9\% to 59.5\%) based on three studies. Most commonly identified pathogens were Staphylococcus aureus and

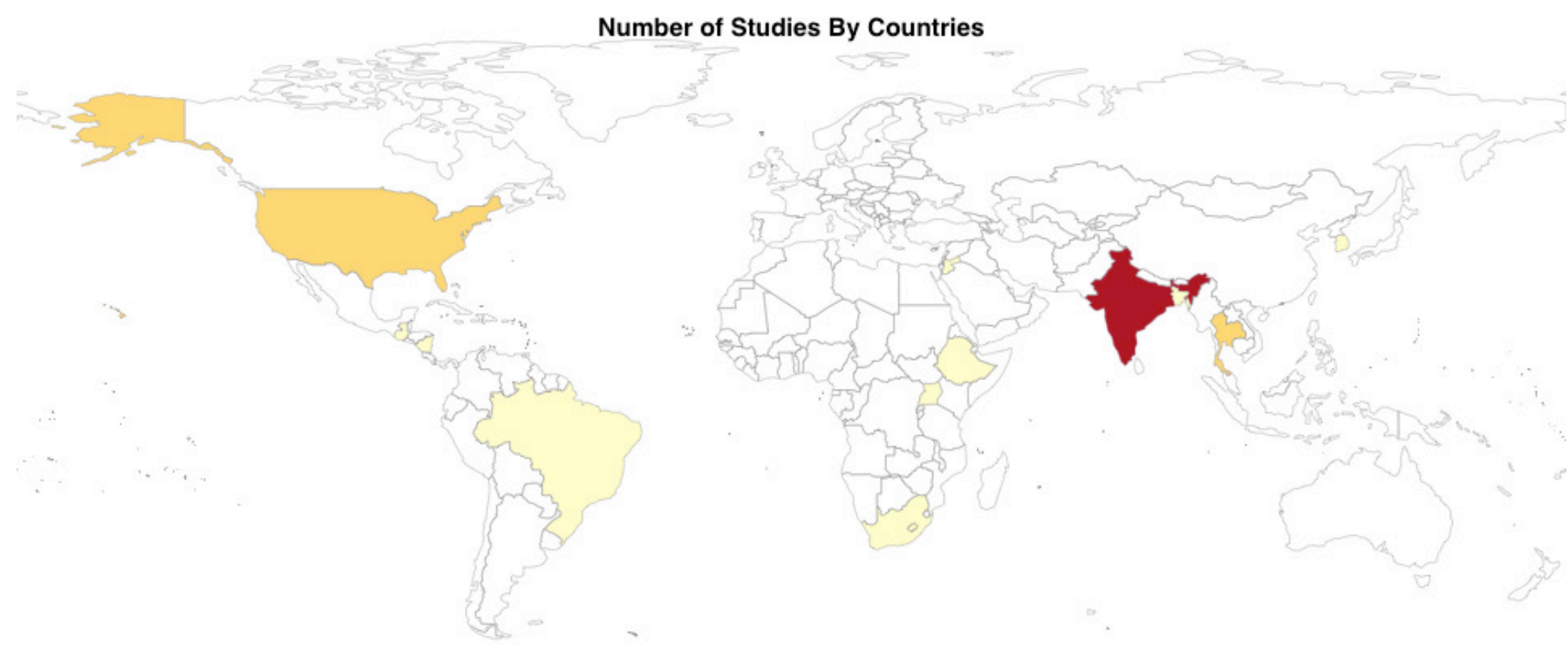

Number of Studies

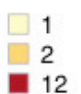

Figure 2 Number of studies on neonatal sepsis incidence (early-onset and late-onset sepsis combined) included per country. 
EOS

Chacko | 2000 - 2001

Goulart | 2004 - 2004

Investigators of the DeNIS collaboration | 2011 - 2014

Kiatchoosakun| 2012 - 2013

Panigrahi | 2002 - 2005

Raihana | 2013 - 2015

Schrag | 2004 - 2007

Shin|1997 - 1997

Shin | 1998 - 1998

Shin | 1999 - 1999

Swarnkar | 2008 - 2010

Turner | 2009 - 2012

Random effects model

Heterogeneity: $I^{2}=100 \%, \tau^{2}=0.9737, p=0$

LOS

Chacko | 2000 - 2001

Investigators of the DeNIS collaboration | 2011 - 2014

Panigrahi | 2002 - 2005

Schrag | 2004 - 2007

Shin| 1997 - 1997

Shin | 1998 - 1998

Shin|1999-1999

Random effects model

Heterogeneity: $I^{2}=99 \%, \tau^{2}=0.5507, p<0.01$

EOS \& LOS

Bang | 1995 - 1996

Bang | 1996 - 2003

Bartlett | 1988 - 1989

Chacko | 2000 - 2001

Ghiorghis| 1992 - 1993

Hartman | 2000 - 2000

Hartman | 2005 - 2005

Investigators of the DeNIS collaboration | 2011 - 2014

Kaushik|..-

Kayom | 2012 - 2012

Khatua | $1982-1983$

López | 2009 - 2009

Martin | 1994 - 2002

Mondal| 1988 - 1989

Nazer | 1977 - 1977

Nazer | 1978 - 1978

Niswade | 2006 - 2007

Nnpd Study Group | 2000 - 2000

Panigrahi | 2002 - 2005

Pavan Kumar | 2013 - 2015

Schrag | 2004 - 2007

Shin| 1997 - 1997

Shin| 1998 - 1998

Shin | 1999 - 1999

Watson | 1995 - 1995

Random effects mode

Heterogeneity: $I^{2}=100 \%, \tau^{2}=1.0755, p=0$

1743

1034
88636

11731

12622

12622
29172

8129

7770

7986

8012

3574

4173

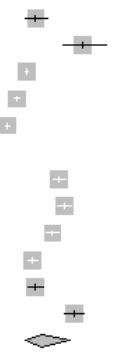

$2065.40 \quad$ [ $1450.67 ; 2848.01]$

5029.01 [ $3778.38 ; 6542.88]$

$1524.21 \quad[1444.58 ; 1607.04]$

878.02 [ 717.21; 1063.85]

301.06 [ 213.13; 413.00]

14126.56 [13728.75; 14531.53]

3555.17 [ $3163.22 ; 3980.86]$

3861.00 [3443.57; 4313.49]

$3117.96 \quad[2747.70 ; 3522.93]$

1847.23 [ $1563.76 ; 2166.44]$

2014.55 [ 1579.52; 2530.34]

4481.19 [3873.56; 5153.52$]$

2469.48 [1423.97; 4249.54]

$1663.80 \quad$ [ $1117.03 ; 2380.81]$

657.75 [ $605.59 ; 713.17]$

3794.96 [ $3468.35 ; 4143.09$ ]

418.26 [ 289.82; 583.98]

682.11 [ $511.35 ; 891.28]$

$751.31 \quad$ [ $573.81 ; 966.05]$

$723.91 \quad$ [ 550.14; 934.83 ]

945.98 [ $543.50 ; 1641.59]$

130

552

16
65

225

4932

8982
1934

1934

62
35
92

92
1286

1286

600

50
5

17

96
899

1899
517

517
107

323

353

309

206

2280

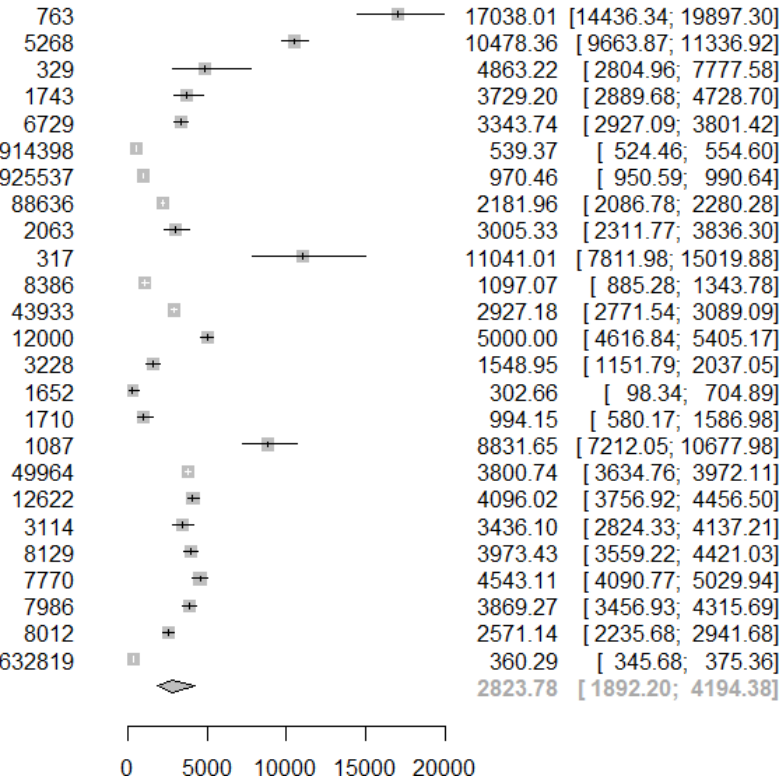

$0 \quad 5000 \quad 10000 \quad 1500020000$

Figure 3 Incidence of neonatal sepsis (EOS, late-onset sepsis, and EOS/LOS combined) per 100000 live births by sepsis type. EOS, early-onset sepsis; LOS, late-onset sepsis.

Klebsiella spp (online supplemental appendix table 3). Results on antimicrobial resistance, which was reported in 3 out of 16 studies, are presented in online supplemental appendix table 3.

\section{DISCUSSION}

Our estimates confirm that neonatal sepsis is an important contributor to neonatal morbidity worldwide, with a higher burden in LMICs. Based on the available evidence of 26 studies from 14 countries, we found an incidence of 2824 neonatal sepsis cases per 100000 live births in the overall time frame. Mortality was $17.6 \%$. The incidence estimate was 1.4 -fold higher in the past decade (2009-2018, 3930/100 000 live births), but this estimate was exclusively relying on LMIC data, which might be one reason for the higher incidence. No recent data from HIC could be identified, indicating gaps in epidemiological research on neonatal sepsis all over the world. The overall incidence and mortality for neonatal sepsis was higher than the estimates for EOS and LOS. This could be explained by inclusion of different studies in the three meta-analyses. Incidence of EOS was higher in risk groups, such as LBW, VLBW and preterm infants. In our analyses, the observed population-level incidence of neonatal sepsis was more than four times higher in community-based compared with hospital-based studies. This may reflect high rates of mothers delivering outside a healthcare facility without a skilled birth attendant, unsterile cord care practices, reduced access to healthcare facilities and lower care seeking behaviours ${ }^{40}$ in LMICs, where the included communitybased studies originated from. On the other hand, hospitalisation, improved survival of preterm newborns and use of invasive devices increase the incidence of nosocomial LOS cases, especially in HIC. ${ }^{4142}$

Our most recent results of neonatal sepsis incidence in the past decade (2009-2018) indicate an approximately four times higher number of global neonatal sepsis cases than the GBD estimates (3930 vs 937 cases per 100 000), likely due to different methodologies, such as varying case definitions, data collection methods 
Original research

Table 1 Meta-analysis estimates and heterogeneity

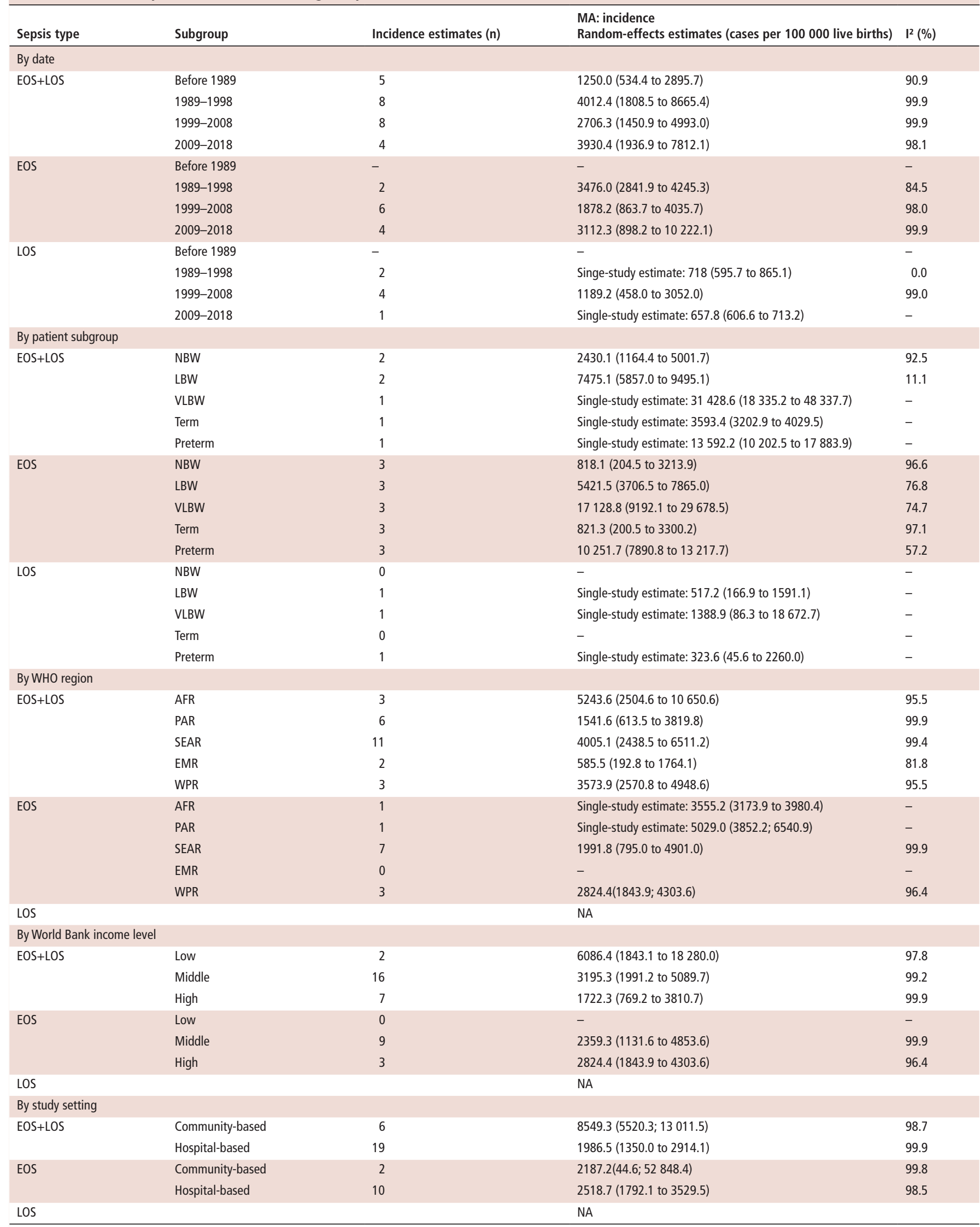

AFR, African Region; EMR, Eastern Mediterranean Region; EOS, early-onset sepsis; LBW, low birth weight; LOS, late-onset sepsis; MA, meta-analysis; NA, not applicable; NBW, normal birth weight; PAR, Region of the Americas; SEAR, South-East Asia Region; VLBW, very low birth weight; WPR, Western Pacific Region. 
and data inputs from different countries. Extrapolations from our findings must be interpreted with caution since the included studies may not be representative of the global population and carry a moderate to high risk of bias. Contrary to most studies that we included in our review, cases of pneumonia are captured in the GBD modelling of pneumonia as a separate entity, but not as neonatal sepsis cases, ${ }^{3}$ which is a major methodological difference. Furthermore, the GBD study used more recent data to compute estimates for the year 2017, while our most recent estimates refer to the past decade (2009-2018). ${ }^{3}$ The recent GBD study, which used another methodology to assess sepsisassociated deaths and hospital incidence of sepsis, estimated 20.3 million incident sepsis cases in children aged $<5$ years per year. ${ }^{43}$ The authors did not present specific data on neonatal sepsis. However, given that neonates account for approximately two out of three sepsis cases in children aged $<5$ years, ${ }^{17}$ the estimated incidence is likely higher than those in previous GBD studies.

Approximately one-third of infections were culture-proven with S. aureus and Klebsiella spp as the most common causative pathogens. However, in most studies that reported aetiology, the assessments of causative pathogens were based on only a low number of isolates or originated from single-centre studies. A cautious interpretation of these results is therefore necessary, other systematic reviews with a scope on aetiology and antibiotic susceptibility could compile more evidence. ${ }^{44}{ }^{45}$ Susceptibility testing results were reported too seldomly and may be outdated, and the data are too heterogeneous to draw conclusions.

The extended search strategy identified 17 additional studies from LMICs and 1 additional study from an HIC. In general, systematic reviews that lack information from LMICs may benefit from a broader search strategy but inclusion of specific LMIC country names, and filters for LMIC as proposed by the EPOC group. We included data from seven community-based studies that followed up a large number of newborns in partly rural regions by trained village health workers/teams and are of particular value to better understand the neonatal sepsis epidemiology in remote and rural community settings in low-income countries.

Major knowledge gaps on the population-based epidemiology of neonatal sepsis remain in most countries, particularly, but not exclusively, in LMICs. Reasons for this may be the lack of a robust research infrastructure, formal healthcare systems ${ }^{46}$ or prioritisation of other important healthcare issues. Furthermore, several large observational studies that target severe neonatal infections did not meet our inclusion criteria either due of their scope or the inclusion criteria.

There are limitations of our study. First, the studies we included may not be representative of the global population for several reasons. Data inputs were from 14 countries from five WHO regions; thus information on the majority of countries and the European WHO region was not included. Numerous studies only reported the incidence of NICU-treated or hospital-treated neonatal sepsis, which may underestimate the incidence of neonatal sepsis by excluding diseased infants outside the hospital setting. This is of particular relevance in LMICs, where a considerable number of births occur outside the hospital ${ }^{47}$ and healthcare seeking for sick neonates may be low. ${ }^{40}$ Furthermore, most hospital-based studies were performed in tertiary care hospitals, which deliver higher quality of care compared with community hospitals, where a large number of neonates may seek care in some contexts. Second, we cannot judge on temporal trends as the number of studies in the respective time strata was low. Thus, differences in incidence estimates depending on the years of observation may be also explained by the fact that studies originated from different regions and used study designs with limited comparability. Third, the included studies were at moderate to high risk of bias, mainly due to the aforementioned lack of representativeness and a reliable case definition. Fourth, we observed high between-study heterogeneity, possibly due to differing study designs, settings and observation periods. The pooled estimates include population-level estimates from community-based studies, as well as NICU-based and hospital-based studies from facilities of different levels of care. General access, capacities and admission policies may differ between NICUs and countries. Heterogeneity may also be driven by different definitions. Applied sepsis definitions are mostly adaptations of the clinical consensus criteria, ${ }^{9}$ such as when laboratory testing was unavailable ${ }^{31}$ or relied on selected clinical symptoms of neonatal sepsis. Currently, there is no sepsis definition for the neonatal age group in line with the current sepsis definition, ${ }^{48}$ which can be applied in all resource settings and also in preterm neonates. That leads to wide variations of existing neonatal sepsis definitions ${ }^{49}$ and hampers the comparability of sepsis epidemiology studies. In our review, we accepted a wide range of definitions, including those based on clinical criteria, to not exclude studies from LMICs. We pooled estimates from all included studies to gather all available evidence on the epidemiology of neonatal sepsis. Fifth, the underlying causes of death in these neonates were not reported except in one of the included studies, which concluded a sepsisattributable mortality rate of $24 \%$. The remaining studies referred to the proportion of patients with neonatal sepsis who died, but the cause of death was not specified. Finally, while our systematic review and pooled estimates consider only live births, globally, one out of four stillbirths are attributed to infection, ${ }^{50}$ adding substantially to the overall burden of perinatal deaths that are due to sepsis.

The limitations identified by our study highlight the need to increase and improve epidemiological research on neonatal sepsis. A crucial prerequisite is the establishment of a harmonised definition and validated diagnostic criteria for neonatal sepsis applicable in all resource settings and in preterm neonates. Furthermore, a standardisation of study designs and reporting, for example, in line with the Strengthening the Reporting of Observational Studies in Epidemiology for Newborn Infection checklist, is important. ${ }^{51}$ Further investments in epidemiological research infrastructure and capacity in both LMICs and HICs are needed in order to improve surveillance of neonatal sepsis, particularly in communitybased designs. Having robust methodologies to measure the burden of neonatal sepsis will be extremely useful to assess the effect of urgently needed interventions to prevent it. Indeed, a lot needs to be done yet to prevent infections in neonates and to reduce the frequency of their evolution to sepsis complications. The WHO issued recommendations on key prevention and control measures both in the community and hospital setting and improved early recognition and timely and appropriate treatment of neonatal sepsis, as well as recommendations for early essential newborn care. ${ }^{52-55}$ This includes, among others, exclusive breast feeding, skin-to-skin contact with the mother from birth, the empirical antibiotic treatment of neonates with signs of severe infection/sepsis (eg, fast breathing) and education of families on the recognition of signs of neonatal sepsis by trained community health workers. ${ }^{545}$ Given that approximately one quarter of neonatal deaths is related to infection and sepsis, progress in reducing the burden of neonatal sepsis is critical to reduce global child mortality and of high public health relevance. 


\section{Author affiliations}

${ }^{1}$ Center for Sepsis Control and Care, Jena University Hospital, Jena, Germany ${ }^{2}$ Institute of Infectious Diseases and Infection Control, Jena University Hospital, Jena, Germany

${ }^{3}$ Department of Infectious Disease Epidemiology, Robert Koch Institute, Berlin, Berlin, Germany

${ }^{4}$ Postgraduate Training for Applied Epidemiology (PAE), Robert Koch Institute, Berlin, Germany

${ }^{5}$ European Programme for Intervention Epidemiology Training (EPIET), European Centre for Disease Prevention and Control (ECDC), Stockholm, Sweden ${ }^{6}$ Infection Prevention and Control Hub, Integrated Health Services, World Health Organization HQ, Geneva, GE, Switzerland

Institute of General Practice and Family Medicine, Jena University Hospital, Jena, Germany

${ }^{8}$ University of British Columbia and British Columbia Children's Hospital, Vancouver, British Columbia, Canada

Institute for Medical Statistics, Computer Science and Data Science, Jena University Hospital, Jena, Germany

${ }^{10}$ Department of Anesthesiology and Operative Intensive Care Medicine (CCM, CVK), Charité Universitätsmedizin Berlin, corporate member of Freie Universität Berlin, Humboldt-Universität zu Berlin, Berlin, Germany

Acknowledgements We thank all authors who provided further details and clarifications of their studies. We thank Liuqing Tong for providing language support for a Chinese publication and Christian Winter, Norman Rose, as well as Angelina Taylor for their valuable comments.

Contributors CF, FR, AC, BA, NK, KR and TE designed the study. CF, FR, RH, MT and YS conducted the literature review, and performed data extraction and the risk of bias assessment. FR performed the statistical analysis. PS and RM advised on the statistical analyses. CF and FR drafted the manuscript. All authors revised the review for important intellectual content and contributed to the writing of the manuscript.

Funding The WHO provided funding for this study and contributed to the study design, data extraction and interpretation, as well as writing of the manuscript.

Disclaimer The opinions expressed in this Article are those of the authors and do not reflect the official position of WHO. WHO takes no responsibility for the information provided or the views expressed in this article.

Map disclaimer The depiction of boundaries on this map does not imply the expression of any opinion whatsoever on the part of BMJ (or any member of its group) concerning the legal status of any country, territory, jurisdiction or area or of its authorities. This map is provided without any warranty of any kind, either express or implied.

Competing interests CF received grants from the German Federal Ministry of Education and Research (BMBF) via the Center for Sepsis Control and Care (FKZ: $01 E 01002$ and 01E01502) and from the Innovation Funds of the German Government (FKZ 01VSF17010). The other authors declared no competing interests.

Patient consent for publication Not required.

Provenance and peer review Not commissioned; externally peer reviewed.

Data availability statement Data are available upon reasonable request. The datasets analysed during the current study are available from the corresponding author on reasonable request. The software codes used during the current study are available in online supplemental file 2.

Supplemental material This content has been supplied by the author(s). It has not been vetted by BMJ Publishing Group Limited (BMJ) and may not have been peer-reviewed. Any opinions or recommendations discussed are solely those of the author(s) and are not endorsed by BMJ. BMJ disclaims all liability and responsibility arising from any reliance placed on the content. Where the content includes any translated material, BMJ does not warrant the accuracy and reliability of the translations (including but not limited to local regulations, clinical guidelines, terminology, drug names and drug dosages), and is not responsible for any error and/or omissions arising from translation and adaptation or otherwise.

Open access This is an open access article distributed in accordance with the Creative Commons Attribution 4.0 Unported (CC BY 4.0) license, which permits others to copy, redistribute, remix, transform and build upon this work for any purpose, provided the original work is properly cited, a link to the licence is given, and indication of whether changes were made. See: https://creativecommons.org/ licenses/by/4.0/.

\section{ORCID iDs}

Carolin Fleischmann http://orcid.org/0000-0002-1104-3191

Felix Reichert http://orcid.org/0000-0003-3205-3513

Alessandro Cassini http://orcid.org/0000-0002-7973-6445

Robby Markwart http://orcid.org/0000-0003-0338-3433

Marc Tröndle http://orcid.org/0000-0002-2364-8112
Niranjan Kissoon http://orcid.org/0000-0001-8847-9973

Peter Schlattmann http://orcid.org/0000-0001-7420-7707

Konrad Reinhart http://orcid.org/0000-0002-8012-6548

\section{REFERENCES}

1 Singer M, Deutschman CS, Seymour CW, et al. The third International consensus definitions for sepsis and septic shock (Sepsis-3). JAMA 2016;315:801-10.

2 Fleischmann-Struzek C, Goldfarb DM, Schlattmann P, et al. The global burden of paediatric and neonatal sepsis: a systematic review. Lancet Respir Med 2018:6:223-30.

3 GBD 2017 Disease and Injury Incidence and Prevalence Collaborators. Global, regional, and national incidence, prevalence, and years lived with disability for 354 diseases and injuries for 195 countries and territories, 1990-2017: a systematic analysis for the global burden of disease study 2017. Lancet 2018;392:1789-858.

4 GBD 2017 Causes of Death Collaborators. Global, regional, and national agesex-specific mortality for 282 causes of death in 195 countries and territories, 1980-2017: a systematic analysis for the global burden of disease study 2017. Lancet 2018;392:1736-88.

5 GBD 2013 Mortality and Causes of Death Collaborators. Global, regional, and national age-sex specific all-cause and cause-specific mortality for 240 causes of death, 1990-2013: a systematic analysis for the global burden of disease study 2013. Lancet 2015;385:117-71.

6 Nwankwor OC, McKelvie B, Frizzola M, et al. A national survey of resources to address sepsis in children in tertiary care centers in Nigeria. Front Pediatr 2019;7:234.

7 Ranjeva SL, Warf BC, Schiff SJ. Economic burden of neonatal sepsis in sub-Saharan Africa. BMJ Glob Health 2018;3:e000347.

8 Cochrane effective practice and organisation of care (EPOC) group. LMIC filters. Available: https://epoc.cochrane.org/lmic-filters

9 Goldstein B, Giroir B, Randolph A, et al. International pediatric sepsis consensus conference: definitions for sepsis and organ dysfunction in pediatrics. Pediatr Crit Care Med 2005:6:2-8.

10 Bone RC, Balk RA, Cerra FB, et al. Definitions for sepsis and organ failure and guidelines for the use of innovative therapies in sepsis. The ACCP/SCCM consensus conference Committee. American College of chest Physicians/Society of critical care medicine. Chest 1992;101:1644-55.

11 Levy MM, Fink MP, Marshall JC, et al. 2001 SCCM/ESICM/ACCP/ATS/SIS international sepsis definitions conference. Crit Care Med 2003;31:1250-6.

12 Seale AC, Blencowe H, Zaidi A, et al. Neonatal severe bacterial infection impairment estimates in South Asia, sub-Saharan Africa, and Latin America for 2010. Pediatr Res 2013;74 Suppl 1:73-85

13 Hoy D, Brooks P, Woolf A, et al. Assessing risk of bias in prevalence studies: modification of an existing tool and evidence of interrater agreement. J Clin Epidemio 2012;65:934-9.

14 Chacko B, Sohi I. Early onset neonatal sepsis. Indian J Pediatr 2005;72:23-6.

15 Ghiorghis B. Neonatal sepsis in Addis Ababa, Ethiopia: a review of 151 bacteremic neonates. Ethiop Med J 1997;35:169-76.

16 Goulart AP, Valle CF, Dal-Pizzol F, et al. [Risk factors for early-onset neonatal sepsis in Brazilian public hospital short-title: early-onset neonatal sepsis]. Rev Bras Ter Intensiva 2006;18:148-53.

17 Hartman ME, Linde-Zwirble WT, Angus DC, et al. Trends in the epidemiology of pediatric severe sepsis*. Pediatr Crit Care Med 2013;14:686-93.

18 Investigators of the Delhi Neonatal Infection Study (DeNIS) collaboration. Characterisation and antimicrobial resistance of sepsis pathogens in neonates born in tertiary care centres in Delhi, India: a cohort study. Lancet Glob Health 2016:4:e752-60.

19 Kaushik SL, Parmar VR, Grover N, et al. Neonatal sepsis in hospital born babies. J Commun Dis 1998:30:147-52.

20 Khatua SP, Das AK, Chatterjee BD, et al. Neonatal septicemia. Indian J Pediatr 1986:53:509-14.

21 Kiatchoosakun P, Jirapradittha J, Sirikarn P, et al. Early-Onset neonatal sepsis and antibiotic use in northeast Thailand. Am J Perinatol 2019:36:1295-303.

22 López S, Wong Y, Urbina L, et al. Quality in practice: preventing and managing neonatal sepsis in Nicaragua. Int J Qual Health Care 2013;25:599-605.

23 Martin TC, Adamson J, Dickson T, et al. Does group B streptococcal infection contribute significantly to neonatal sepsis in Antigua and Barbuda? West Indian Med J 2007:56:498-501.

24 Mondal GP, Raghavan M, Bhat BV, et al. Neonatal septicaemia among inborn and outborn babies in a referral hospital. Indian J Pediatr 1991:58:529-33.

25 Nazer H. Neonatal septicaemia at the Jordan university hospital. J Trop Pediatr 1981:27:199-204.

26 Nnpd Study Grp. Neonatal sepsis in India: bacterial isolates and their antimicrobial sensitivity at leading tertiary care institutions of India. Pediatric Research 2002;1:298A-A.

27 Pavan Kumar DV, Mohan J, Rakesh PS, et al. Bacteriological profile of neonatal sepsis in a secondary care hospital in rural Tamil Nadu, southern India. J Family Med Prim Care 2017;6:735-8. 
28 Schrag SJ, Cutland CL, Zell ER, et al. Risk factors for neonatal sepsis and perinatal death among infants enrolled in the prevention of perinatal sepsis trial, Soweto, South Africa. Pediatr Infect Dis J 2012;31:821-6.

29 Shin Y-J, Ki M, Foxman B. Epidemiology of neonatal sepsis in South Korea. Pediatr Int 2009;51:225-32.

30 Swarnkar KS. A study of early onset neonatal sepsis with special reference to sepsis screening parameters in a tertiary care centre of rural India. Int J Infect Dis 2012;10.

31 Turner C, Turner P, Hoogenboom G, et al. A three year descriptive study of early onset neonatal sepsis in a refugee population on the Thailand Myanmar border. BMC Infect Dis 2013;13:601.

32 Watson RS, Carcillo JA, Linde-Zwirble WT, et al. The epidemiology of severe sepsis in children in the United States. Am J Respir Crit Care Med 2003;167:695-701.

33 Bang AT, Bang RA, Baitule S, et al. Burden of morbidities and the unmet need for health care in rural neonates--a prospective observational study in Gadchiroli, India. Indian Pediatr 2001;38:952-65.

34 Bang AT, Bang RA, Stoll BJ, et al. Is home-based diagnosis and treatment of neonatal sepsis feasible and effective? seven years of intervention in the Gadchiroli field trial (1996 to 2003). J Perinatol 2005;25 Suppl 1:S62-71.

35 Bartlett AV, Paz de Bocaletti ME, Bocaletti MA. Neonatal and early postneonatal morbidity and mortality in a rural Guatemalan community: the importance of infectious diseases and their management. Pediatr Infect Dis J 1991;10:752-7.

36 Kayom VO, Mugalu J, Kakuru A, et al. Burden and factors associated with clinical neonatal sepsis in urban Uganda: a community cohort study. BMC Pediatr 2018;18:355

37 Niswade A, Zodpey SP, Ughade S, et al. Neonatal morbidity and mortality in tribal and rural communities in central India. Indian J Community Med 2011;36:150-8.

38 Panigrahi P, Chandel DS, Hansen NI, et al. Neonatal sepsis in rural India: timing, microbiology and antibiotic resistance in a population-based prospective study in the community setting. J Perinatol 2017;37:911-21.

39 Raihana S, Rahman MM, Tahsina T. Timing of initiation of breastfeeding and EARLY-NEWBORN sepsis: evidence from rural Bangladesh. Annals of Nutrition and Metabolism 2017:71:563.

40 Herbert HK, Lee ACC, Chandran A, et al. Care seeking for neonatal illness in low- and middle-income countries: a systematic review. PLoS Med 2012:9:e1001183.

41 Dong Y, Speer CP. Late-Onset neonatal sepsis: recent developments. Arch Dis Child Fetal Neonatal Ed 2015:100:F257-63.
42 Bizzarro MJ, Raskind C, Baltimore RS, et al. Seventy-Five years of neonatal sepsis at Yale: 1928-2003. Pediatrics 2005;116:595-602.

43 Rudd KE, Johnson SC, Agesa KM, et al. Global, regional, and national sepsis incidence and mortality, 1990-2017: analysis for the global burden of disease study. Lancet 2020;395:200-11

44 Downie L, Armiento R, Subhi R, et al. Community-acquired neonatal and infant sepsis in developing countries: efficacy of WHO's currently recommended antibiotics-systematic review and meta-analysis. Arch Dis Child 2013;98:146-54.

45 Lubell Y, Ashley EA, Turner C, et al. Susceptibility of community-acquired pathogens to antibiotics in Africa and Asia in neonates--an alarmingly short review. Trop Med Int Health 2011:16:145-51.

46 Diaz JV, Riviello ED, Papali A, et al. Global critical care: moving forward in resourcelimited settings. Ann Glob Health 2019;85. doi:10.5334/aogh.2413. [Epub ahead of print: 22 Jan 2019]

47 Montagu D, Yamey G, Visconti A, et al. Where do poor women in developing countries give birth? A multi-country analysis of demographic and health survey data. PLoS One 2011;6:e17155.

48 Schlapbach LJ, Kissoon N. Defining pediatric sepsis. JAMA Pediatr 2018;172:313-4.

49 Wynn JL. Defining neonatal sepsis. Curr Opin Pediatr 2016;28:135-40.

50 McClure EM, Dudley DJ, Reddy UM, et al. Infectious causes of stillbirth: a clinical perspective. Clin Obstet Gynecol 2010;53:635-45.

51 Fitchett EJA, Seale AC, Vergnano S, et al. Strengthening the reporting of observational studies in epidemiology for newborn infection (STROBE-NI): an extension of the STROBE statement for neonatal infection research. Lancet Infect Dis 2016;16:e202-13.

52 Pittet D, Allegranzi B. Preventing sepsis in healthcare - 200 years after the birth of Ignaz Semmelweis. Euro Surveill 2018;23.

53 Storr J, Twyman A, Zingg W, et al. Core components for effective infection prevention and control programmes: new who evidence-based recommendations. Antimicrob Resist Infect Control 2017;6:6

54 World Health Organization. Who recommendations on newborn health. guidelines Approved by the who guidelines review Committee, 2017. Available: https://www. who.int/maternal_child_adolescent/documents/newborn-health-recommendations/ en/

55 World Health Organization ROftWP. Early essential newborn care : clinical practice pocket guide, 2014. Available: https://apps.who.int/iris/bitstream/handle/10665/ 208158/9789290616856 eng.pdf 